\title{
Numerical Analysis of Urea Decomposition with Static Mixers in Marine SCR System
}

\author{
Cheolyong Choi, Yonmo Sung, Gyung Min Choi, and Duck Jool Kim
}

\begin{abstract}
A numerical simulation for urea decomposition into ammonia with static mixers was performed for marine selective catalytic reduction. Three types of mixer were considered to improve urea into ammonia conversion rate. The effect of each mixer such as pressure drop, particle residence time, and uniform distribution of urea water solution was investigated using three dimensional CFD code. Using mixer regardless of a type was improved urea decomposition. Engine performance could be affected by high pressure drop caused by mixer. Therefore, the proper mixer should be chosen in permissible level of pressure drop as well as ammonia conversion rate.
\end{abstract}

Index Terms-Selective catalytic reduction (SCR), static mixer, urea decomposition, urea water solution (UWS).

\section{INTRODUCTION}

The International Maritime Organization's Tier III standards require that marine vessels have to meet reducing $\mathrm{NO}_{\mathrm{x}}$ emissions in 2016 [1]. It is necessary to satisfy the regulation for ships built after the effective date of the regulation. The Selective Catalytic Reduction (SCR) is used to reduce nitrogen oxides $\left(\mathrm{NO}_{\mathrm{x}}\right)$ emissions and commonly used for many industries such as power generation boilers, diesel engine vehicles, and marine vessels. A reducing agent is injected into a exhaust pipe, and removes the $\mathrm{NO}_{\mathrm{x}}$ emissions through several steps. As a reducing agent, urea water solution (UWS) is preferred to gaseous $\mathrm{NH}_{3}$ because of its toxicity and storage problems. UWS is decomposed and generates gaseous $\mathrm{NH}_{3}$. Urea decomposition processes are divided into thermolysis and hydrolysis, and they can be expressed as follows:

$$
\begin{aligned}
& \left(\mathrm{NH}_{2}\right)_{2} \mathrm{CO} \rightarrow \mathrm{NH}_{3}+\mathrm{HNCO} \\
& \mathrm{HNCO}+\mathrm{H}_{2} \mathrm{O} \rightarrow \mathrm{NH}_{3}+\mathrm{CO}_{2}
\end{aligned}
$$

$\mathrm{NO}_{\mathrm{x}}$ reduction reaction as well as urea decomposition processes mostly takes place in a catalyst filter. It is needed to use a mixer which blends the flow and UWS, and is also expected to expedite the reactions. There are many researches about selecting a mixer type and urea decomposition through experiment and simulation. Thakur et al. gave guidelines for the selection of static mixers [2]. Zheng et al. was defined the scope of the mixer development for given exhaust flow configurations [3], [4]. They also defined the main causes of

Manuscript received November 24, 2013; revised January 22, 2014. This work was supported by the National Research Foundation of Korea (NRF) grant funded by the Korea government (MEST) through GCRC-SOP.

The authors are with the School of Mechanical Engineering, Pusan National University, Busan, Korea (e-mail: cychoi@pusan.ac.kr; ymsung@pusan.ac.kr; choigm@pusan.ac.kr; djkim@pusan.ac.kr). urea deposits on mixers based on the SCR system that already demonstrated optimized urea mixing and low back pressure. Zhang et al. evaluated the effects of turbulent flow, as well as swirling flow, on $\mathrm{NH}_{3}$ mixing [5]. Sung et al. studied numerically on the relationship between flow mixing characteristics and pressure drop under the different vane angles [1]. Birkhold et al. discussed spatial enthalpy variations due to evaporation, thermolysis, and hydrolysis of UWS, and derived the model to judge different SCR exhaust system configurations with respect to conversion and local distribution of reducing agent [6], [7].

However, there has been relatively insufficient research on urea decomposition under using mixer in the practical marine SCR system. Therefore, the purpose of this study is to evaluate the effect of mixer structure on urea to $\mathrm{NH}_{3}$ conversion rate.

\section{NUMERICAL PROCEDURE}

\section{A. Computational Domain}

To investigate numerically urea decomposition reactions in the SCR system, the computational domain is modeled in three dimensional geometry by using Gambit 2.4.6 as shown in Fig. 1. It is considered three types of mixers to find out effect of mixer structure. Each mixer has 36 vanes and the same angle of $45^{\circ}$, but inclines to different directions as shown in Fig. 2. All vanes also have the same area so as to investigate the effect on pressure drop. Fig. 2(a) is a conventional type of mixer which induces up-and-down flow. Fig. 2(b) is an entire swirl type mixer, and Fig. 2(c) is a partial swirl mixer. In front of the mixer, UWS as a reducing agent is injected into the exhaust pipe. Droplets are spread, mixed with the gas, and decomposed into $\mathrm{NH}_{3}$ by flow characteristics. The SCR reactor is located in the middle of exhaust pipe. It has a square cross-sectional area and is connected with the exhaust pipe. There is a catalyst filter which is assumed as a porous media.

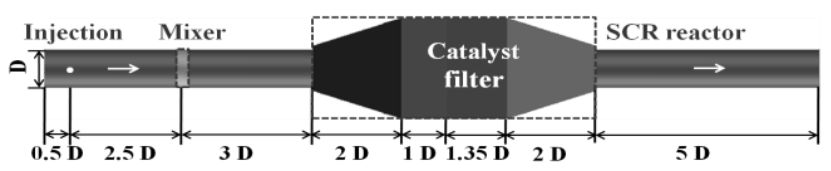

Fig. 1. The computational domain of SCR system.

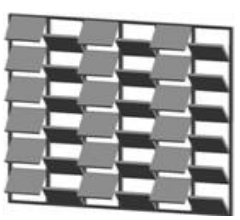

(a)

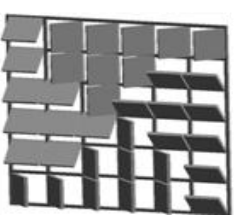

(b)

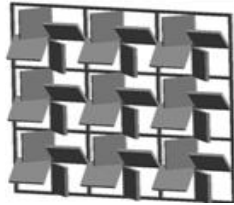

(c)
Fig. 2. Geometry of three types of mixers; (a) up-and-down, (b) entire swirl, (c) partial swirl. 


\section{B. Numerical Models}

As exhaust gas passes the mixers, turbulence is intensified due to induced flow. To predict complicated turbulent flow, it is calculated using a shear-stress transport (SST) $k$ - $\omega$ model which is based on Reynolds-averaged Navier-Stokes (RANS) equation. In this study, the commercial CFD code FLUENT 6.3.26 was used for numerical analysis.

Catalyst filter in the SCR reactor is treated as porous media. Porous media are modeled by the addition of a momentum source term to the standard fluid flow equations. The source term is composed of two parts: a viscous loss term, and an inertial loss term.

$$
S_{i}=-\left\{(\mu / \alpha) v_{i}+C_{2} \rho|v| v_{i}\right\}
$$

where $S_{i}$ is the source term for the $i$ th momentum equation, $|v|$ is the magnitude of the velocity, $\alpha$ is the permeability, and $C_{2}$ is the inertial resistance factor. Table I shows the used coefficients.

The reaction model is validated with the experimental data which was conducted by Kim et al. [8]. As shown in Fig. 3, it shows a similar tendency with the experimental data although it does not match with a low velocity condition. It means that this model cannot use at a low velocity condition below 9.1 $\mathrm{m} / \mathrm{s}$. This reaction model is used at high velocity condition in this study. In order to investigate effects of mixers, it is divided into four cases as shown in Table II. Case 1 does not include any mixers.

TABLE I: THE COEFFICIENTS FOR POROUS MEDIA MODEL

\begin{tabular}{ccc}
\hline \hline Variable & $1 / \alpha$ & $\mathrm{C}_{2}$ \\
\hline Value & 900,000 & 7.5 \\
\hline \hline
\end{tabular}

TABLE II: ClASSIFIED CASES ACCORDING TO THE MIXER TYPE

\begin{tabular}{cc}
\hline Case & Mixer type \\
\hline 1 & w/o \\
2 & Up-and-down \\
3 & Entire swirl \\
4 & Partial swirl \\
\hline \hline
\end{tabular}

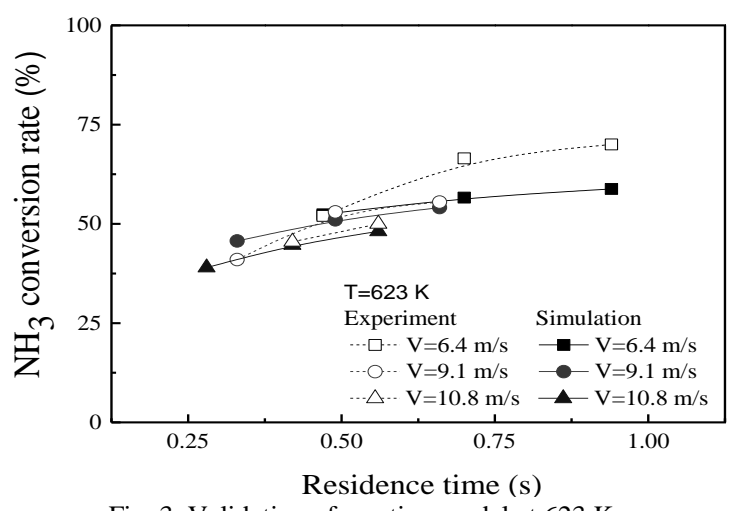

Fig. 3. Validation of reaction model at $623 \mathrm{~K}$.

\section{Initial and Boundary Conditions}

UWS is injected at $0.5 \mathrm{D}$ from inlet. UWS is injected to downstream. In point of interaction between exhaust gas and the injected reducing agent, it is too short for exhaust gas to be developed. Therefore, fully-developed velocity profile is used for initial inlet condition. Exhaust gas is assumed to be composed of $77 \% \mathrm{~N}_{2}$ and $23 \% \mathrm{O}_{2}$ in mass fraction, and have a $15.4 \mathrm{~m} / \mathrm{s}$ as mean velocity and $623 \mathrm{~K}$ as a uniform temperature.
Injected particles are assumed to follow the Rosin-Rammler distribution. Mean diameter of particle is $44 \mu \mathrm{m}$ and spread parameter is 3.5. Cone type spray is adopted, and it has $70^{\circ}$ of spray angle. Spray velocity is similar to the inlet velocity, 15 $\mathrm{m} / \mathrm{s}$, and spray temperature is $300 \mathrm{~K}$.

\section{RESUltS AND DISCUSSION}

The presence of mixer increases pressure drop in the system. Cases 2, 3, and 4 have $80 \%, 75 \%$, and $116 \%$ higher pressure drop compared to case1, respectively. Case 2 and 3 have a similar level of pressure drop, however case 4 has a relatively higher level of pressure drop. Although all mixer vanes have the same area and angle and the inlet velocity is constant, the reason why it takes place differences among cases is a complicated flow disturbance caused by the inclined direction. In point of geometry of mixers, flow caused by the up-and-down type and the entire swirl type mixer meets one different directional flow, whereas flow caused by the partial swirl mixer meets three different directional flow. It is necessary to control the pressure drop by a certain component in the aftertreatment devices, because it can cause a decreasing engine power. It is hard to conclude that case 4 affects the engine power, but it has to be considered when selecting a mixer. It is needed to define a permissible level of pressure drop with respect to an engine specification.

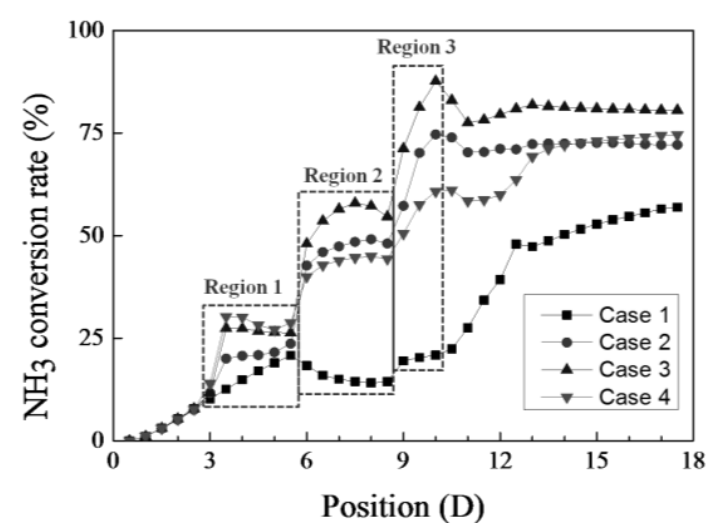

Fig. 4. $\mathrm{NH}_{3}$ conversion rate of each case along streamwise.

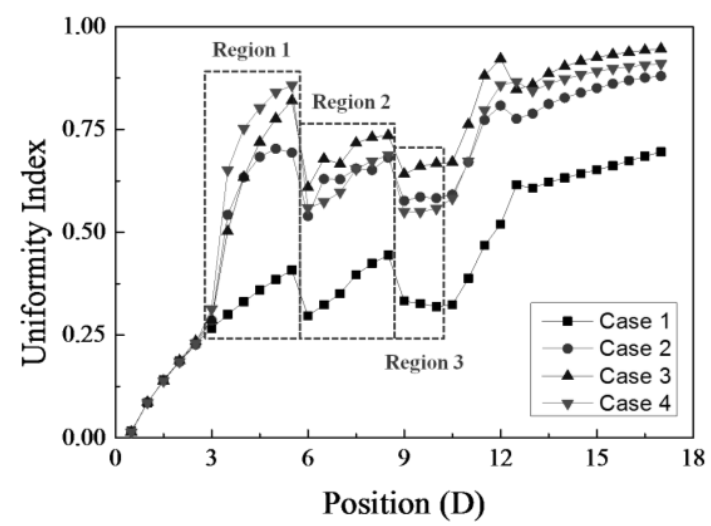

Fig. 5. Uniformity index of $\mathrm{NH}_{3}$ at each case.

Fig. 4 shows the $\mathrm{NH}_{3}$ conversion rate at each position along downstream of SCR system. $\mathrm{NH}_{3}$ conversion rate is defined as the ratio of an amount of $\mathrm{NH}_{3}$ production to the maximum amount of $\mathrm{NH}_{3}$ when UWS is totally converted into $\mathrm{NH}_{3}$. Fig. 5 shows uniformity index of $\mathrm{NH}_{3}$. Uniform distribution of 
$\mathrm{NH}_{3}$ is required to minimize $\mathrm{NH}_{3}$ slip and to maximize de- $\mathrm{NO}_{x}$ efficiency. Uniformity index of $\mathrm{NH}_{3}$ is defined as follow:

$$
U I=1-\frac{1}{2 n} \sum_{i=1}^{n} \frac{\sqrt{\left(c_{i}-\bar{c}\right)^{2}}}{\bar{c}}
$$

where, $c_{i}$ is a local concentration of $\mathrm{NH}_{3}, \bar{c}$ is an average concentration of $\mathrm{NH}_{3}$, and $\mathrm{n}$ is a number of cells. If uniformity index has a value of 1 , it means perfectly distributed.

It seems to be divided into three regions in point of producing $\mathrm{NH}_{3}$. The first region is rear side of mixer before the SCR reactor (3 D-6 D), the second region is front side of catalyst filter in the reactor (6 D-9 D), and the last region is inside of the catalyst filter $(9 \mathrm{D}-10 \mathrm{D})$. As the flow and UWS pass through each region, $\mathrm{NH}_{3}$ conversion rate is dramatically increased in all cases using mixer. In the first region, case 4 has the highest $\mathrm{NH}_{3}$ conversion rate, and case 3 has a slightly smaller value compared to case 4 . From the second region, however, the order of $\mathrm{NH}_{3}$ conversion rate is completely changed. Case 3 has the highest value. In the last region, $\mathrm{NH}_{3}$ conversion rate of all cases is increased because of high pressure in catalyst filter. These results are related to particle distribution.

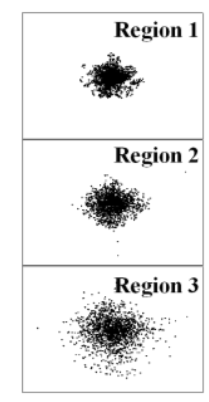

(a) Case 1

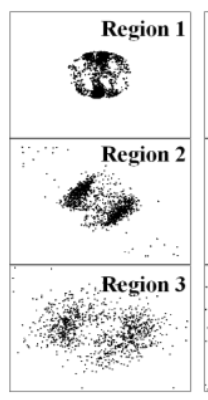

(b) Case 2

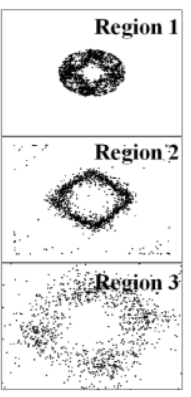

(c) Case 3

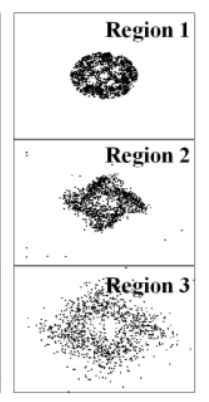

(d) Case 4
Fig. 6. Particle distribution on a cross-sectional area at each region; $3.5 \mathrm{D}$ in region $1,7.5 \mathrm{D}$ in region 2 , and $10 \mathrm{D}$ in region 3.

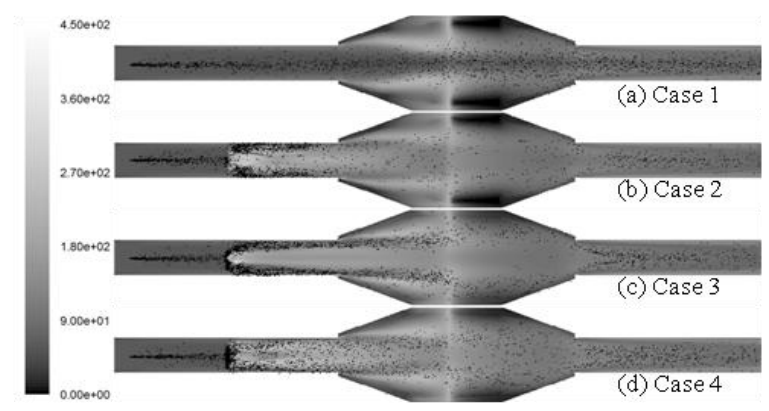

Fig. 7. Turbulence intensity (\%) and particle distribution of each case.

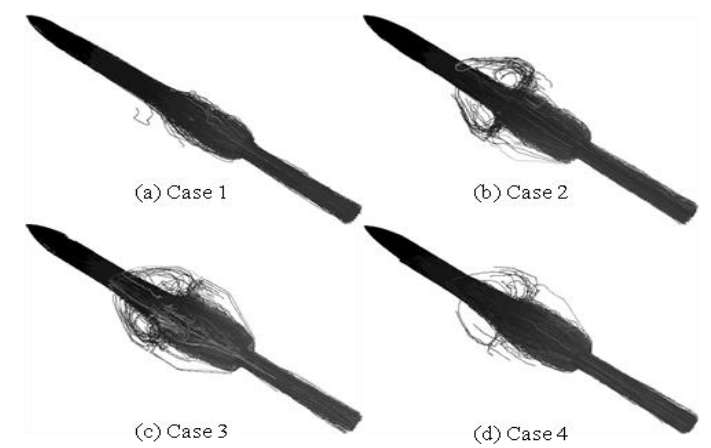

Fig. 8. Isometric view of particle trajectories of each case.

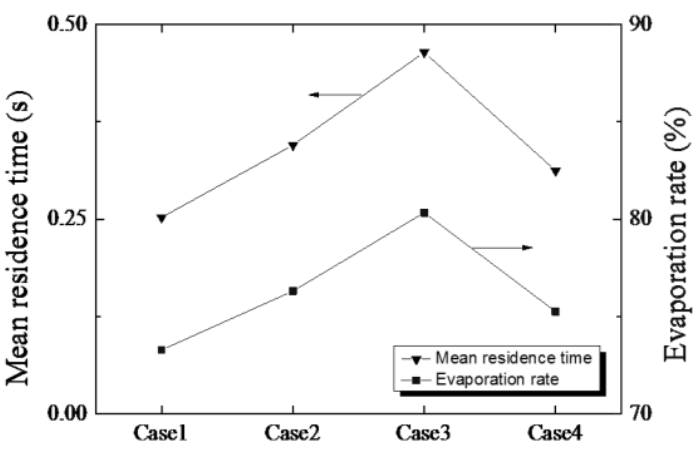

Fig. 9. Mean particle residence time and evaporation rate.

Fig. 6 represents particle distribution of each case at positions $3.5 \mathrm{D}, 7.5 \mathrm{D}$, and $10 \mathrm{D}$. It shows particle motion through each mixer, and particles are located by its flow. In case 1, particles are rarely diffused along downstream. In the first region, particles are distributed to top and bottom side of exhaust pipe in case 2 . In case 3, particles are distributed to radial direction and the center of exhaust pipe becomes empty. However, it shows well distributed particles in case 4. It causes the highest $\mathrm{NH}_{3}$ conversion rate as shown in Fig. 4 because UWS easily decomposes by heat transfer through surface area of particles.

Nevertheless, the uniform distribution in region 1 does not mean fast urea decomposition. It is not good after the particles go into the second region. Exhaust pipe is connected with a square SCR reactor, and it makes sharp edge. Then, this edge makes a high level of turbulent intensity due to flow separation. Although case 4 has the well distributed particles, it does not generate $\mathrm{NH}_{3}$ compared to cases 2 and 3 . In cases 2 and 3, most particles are located in the side wall of exhaust pipe in the first region. As particles move into the second region, it is effectively affected by a high level of turbulence intensity caused by the edge, especially in case 3 , as shown in Fig. 7. Only some parts of particles are affected by the high level of turbulence intensity in case 4. It means that matching particle location with a high turbulence intensity area is important to improve the $\mathrm{NH}_{3}$ conversion rate.

It can be seen widely spread particles in the last region of case 3. It is obvious that case 3 has the highest $\mathrm{NH}_{3}$ conversion rate. At the same position, cases 2 and 3 have a value of $\mathrm{NH}_{3}$ conversion rate. It seems to have a similar particle distribution both cases 2 and 4 in the last region. However, the difference of $\mathrm{NH}_{3}$ conversion rate can be explained by the residence time of particles. Fig. 8 shows the particle trajectories, and Fig. 9 shows mean residence time and the evaporation rate of droplets. Case 1 has the shortest mean residence time and particle recirculation rarely takes place. In case 2, particle motion is less than case 3 although it covers a wide range before the filter. Case 3 has the most vigorous recirculation and the longest mean residence time. Particles tend to spread out strongly to the extended area in regions 2 and 3. On the other hand, only a few particles are recirculated in case 4 . Mean residence time is also decreased evidently. It does not have enough momentum to spread out although it has well distributed particles in region 1 .

Furthermore, it seems that the evaporation rate is proportion to mean residence time as shown in Fig. 9. It is equal to the degree of recirculation and the tendency of $\mathrm{NH}_{3}$ conversion rate. It is concluded that the particle recirculation 
enhances residence time and evaporation rate. Then, the hydrolysis process is expedited, and a large amount of $\mathrm{NH}_{3}$ is generated.

\section{CONCLUSION}

A numerical simulation is performed to investigate the effects of mixer structure on urea to the $\mathrm{NH}_{3}$ conversion rate. The main results are expressed as follows:

1) The SCR system can be divided into three region in point of $\mathrm{NH}_{3}$ conversion rate. The first region is rear side of mixer before the SCR reactor, the second region is front side of catalyst filter in the reactor, and the last region is inside of the catalyst filter.

2) In general, urea decomposition is activated when it is developed the uniform particle distribution. However, it is observed that $\mathrm{NH}_{3}$ conversion rate is improved when the particle distribution is matched with a high turbulence intensity area despite non-uniform distribution.

3) Particle residence time is related to the degree of recirculation and is proportion to the droplet evaporation rate. It affects to urea decomposition directly.

\section{REFERENCES}

[1] Y. M. Sung, C. Y. Choi, T. K. Kim, G. M. Choi, and D. J. Kim, "Effect of geometric structure of static mixers on mixing characteristics and pressure drop in marine SCR applications," presented at International Conference on Multiphase Flow, Jeju, Korea, May 26-31, 2013.

[2] R. K. Thakur, C. Vial, K. D. P. Nigam, E. B. Nauman, and G. Djelveh, "Static mixers in the process industries-a review," Chemical Engineering Research and Design, vol. 81, pp. 787-826, Aug. 2003.

[3] G. Zheng, G. Palmer, G. Salanta, and A. Kotrba, "Mixer development for urea SCR applications," SAE Technical Paper 2009-01-2879, 2009 ,

[4] G. Zheng, A. Fila, A. Kotrba, and R. Floyd, "Investigation of urea deposits in urea SCR systems for medium and heavy duty trucks," SAE Technical Paper 2010-01-1941, 2010.

[5] X. Zhang, M. Romzek, and C. Morgan, "3-D Numerical Study of Mixing Characteristics of NH3 in Front of SCR," SAE Technical Paper 2006-01-3444, 2006.

[6] F. Birkhold, U. Meingast, P. Wassermann, and O. Deutschmann, "Analysis of the injection of urea-water-solution for automotive SCR DeNOx-systems: modeling of two-phase flow and spray/wall-interaction," SAE Technical Paper 2006-01-0643, 2006.
[7] F. Birkhold, U. Meingast, P. Wassermann, and O. Deutschmann, "Modeling and simulation of the Injection of Urea-water-solution for Automotive SCR DeNOx-system," Applied Catalysis B: Environmental, vol. 70, pp. 119-127, 2007.

[8] J. Y. Kim, S. H. Ryu, and J. S. Ha, "Numerical prediction on the characteristics of spray-induced mixing and thermal decomposition of urea solution in SCR system," in Proc. 2004 Fall Technical Conference of the ASME Internal Combustion Engine Division, Long Beach, California, USA, 2004.

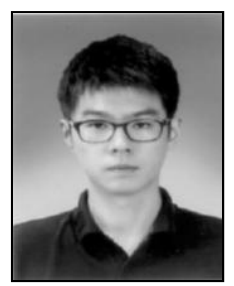

Cheolyong Choi earned BSc in mechanical engineering from Pusan National University, Busan, Korea in 2013.

$\mathrm{He}$ is enrolled as a master student in School of Mechanical Engineering, Pusan National University, Busan, Korea.

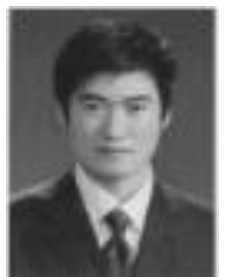

Yonmo Sung earned MSc in mechanical engineering from Pusan National University, Busan, Korea in 2010.

$\mathrm{He}$ is enrolled as a Ph.D. student in School of Engineering, Pusan National University, Busan, Korea.

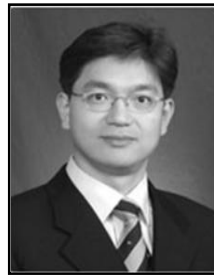

Gyung Min Choi earned Ph.D. in mechanical engineering from Pusan National University, Busan, Korea in 1997 and from Osaka University, Osaka, Japan in 2002.

$\mathrm{He}$ is a professor in School of Mechanical Engineering, Pusan National University, Busan, Korea Prof. Choi is in charge of general affairs for the Korean Society of Combustion.

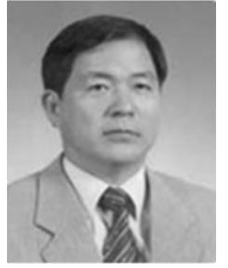

Duck Jool Kim earned Ph.D. in mechanica engineering from Kyungpook National University, Daegu, Korea

$\mathrm{He}$ is a professor in School of Mechanical Engineering, Pusan National University, Busan, Korea Prof. Kim was in charge of a vice-president for the Institute for Liquid Atomization and Spray Systems-Korea. 